\title{
Simulating the diffraction grating reflectivity using effective medium theory
}

\author{
A.A. Goloborodko, N.S. Goloborodko, Ye.A. Oberemok, S.N. Savenkov \\ Taras Shevchenko Kyiv National University, Department of Radiophysics, \\ 64, Volodymyrska str., 01601 Kyiv, Ukraine \\ E-mail:angol@univ.kiev.ua
}

\begin{abstract}
The features of optical radiation interaction with surface of gratings are investigated. The diffraction grating is proposed to be used in the effective medium model to test nanostructured surfaces. This object allows obtaining simultaneous visualization of different spatial frequencies and estimation of both structure and surface features of 3D-objects. It was shown that the effective medium model could be used to predict the reflectivity of nanostructured surfaces by using diffraction gratings with different depths of profile. Computer simulations show that the filling factor of composite systems could be an angle dependent function. So, the correct solution of the inverse problem of finding surface characteristics, such as refraction indices and absorption coefficients, is related with the angle dependence of filling factor.
\end{abstract}

Keywords: diffraction grating, effective medium, refraction index, absorption coefficient.

Manuscript received 15.01.13; revised version received 22.02.12; accepted for publication 19.03.12; published online 25.06.12.

\section{Introduction}

Nowadays, nondestructive, multiple parameters, express detection of structures local features become more necessary with nanotechnologies improvement. For the most widespread layered film structures the main features are: the layer thickness and basical optical features such as refraction index $n$ [1] and absorption coefficient $\kappa$ [2]. It should be mentioned that dependences $n(d)$ and $\kappa(d)$ for nanostructured surfaces, obtained for one wavelength, can vary with the wavelengths change $[3,4]$. Thus, using the value of some parameter from tables or literature data can be inaccurate or can lead to essential errors in diagnosing the other features.

So, the solution of this problem is in determination of a set of bound parameters within a unified method and one measuring system that allow defining a set of features in one measurement. However, currently optical methods that allow simultaneous determination of refraction index, absorption coefficient and thickness for absorbing and nonabsorbing media are developed insufficiently. Widespread angular methods [5] were applied only to bulk samples for determining only two parameters $-n$ and $\kappa$.

Big success in nanostructures investigation was achieved for porous silicon [6], which consists of silicon nanocrystals separated by air. If the characteristic sizes of pores and nanocrystals are much smaller than the irradiation wavelength, then such a semiconductor can be considered as homogeneous optical medium, which has some effective refraction index and absorption coefficient. These characteristics differ from characteristics of bulk samples that form the nanostructure. In this case, the model of effective medium for defining the characteristics of nanostructured objects should be discussed. The electrostatic approximation is frequently used in the model of effective medium for optical problems. This approximation requires both small sizes of the nanoparticles and a short distance between them as compared to the optical wavelength in the medium [7]. 
In the other case, there arises the problem of taking into account the scattering on particles that form the nanocomposite medium and interference of reflected waves. Thus, the aim of this paper was to create the optical scheme suitable for visualization and investigation of polarization characteristics of diffraction grating and effective medium model verification for reflected light characteristics description.

\section{Ellipsometric investigation of ordinary grating}

The diffraction gratings are often used as tested objects for calibration of optical devices. And usage of diffraction gratings with appearance of nanostructured composite materials (inhomogeneity size values of which are about the wavelength $\lambda$ of scanning beam) needs an additional investigation. Thus, the medium model based on diffraction gratings with the period $d=$ $1 \mu \mathrm{m}$ and variable height $h$ from 12 up to $300 \mathrm{~nm}$ with the step $12 \mathrm{~nm}$ are considered in this work. These gratings were produced using the electron beam lithography method on photoresist (electron-sensitive PMMA resist). A reconstructed 3D image received with a scanning electron microscopy (SEM) method is shown in Fig. 1.

The optical scheme shown in Fig. 2 was used for experiment. This scheme includes the probing (Laser, $\mathrm{P}$, L1, L2) and receiving (L3, L4, WP1, WP2, PhD1, $\mathrm{PhD} 2$ ) channels. Coherent radiation from a He-Ne laser with $\lambda=0.63 \mu \mathrm{m}$, which was used as a source, was formed into converging beams by the long-focus system L1 - L2 illuminating the grating entirely. After reflection from the grating, the main maximum in the mirror reflection direction and a great number of secondary orders were observed. This scheme allows suitable observation of diffraction orders from a grating with submicron relief dimensions that were upright spacing from the main order. The photodetectors PhD1 and $\mathrm{PhD} 2$ were used to register the diffraction maximum intensities and were situated on the goniometry arms.

Fig. 3 shows experimental dependences of the intensity inherent to the main and first diffraction orders for gratings with different profile heights in the case of normal incidence.

As one can see, behavior of these dependences shows their anticorrelation and illustrates the energy redistribution between diffraction maxima (from the main to higher orders). Thereby, next calculations require taking into account the intensities redistribution in the maxima with the relief depth changing. In addition, the curves in Fig. 3 lead to the conclusion that there is no interference for gratings with profile depths less than $84 \mathrm{~nm}$, so the model of effective medium may be applied to them. Contribution of the first diffraction orders for a deeper depth of the grating profile becomes more substantial, and the model with effective refraction index requires some corrections for the further theoretical calculations.

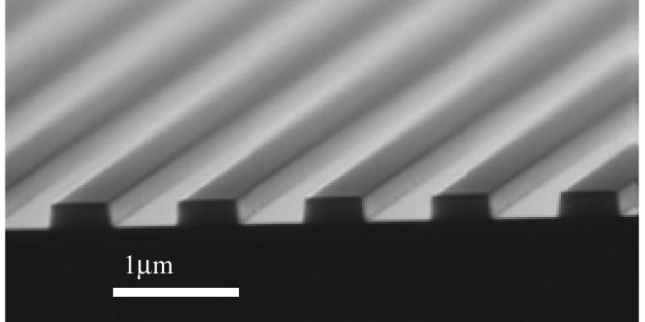

Fig. 1. 3D SEM image of the grating.

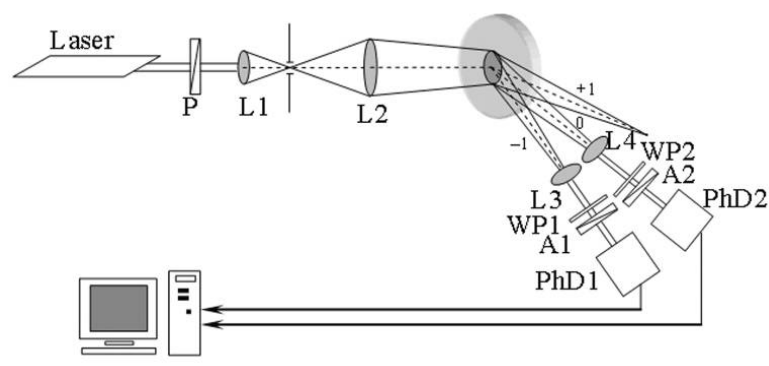

Fig. 2. Optical setup for simultaneous visualization of different spatial frequencies.

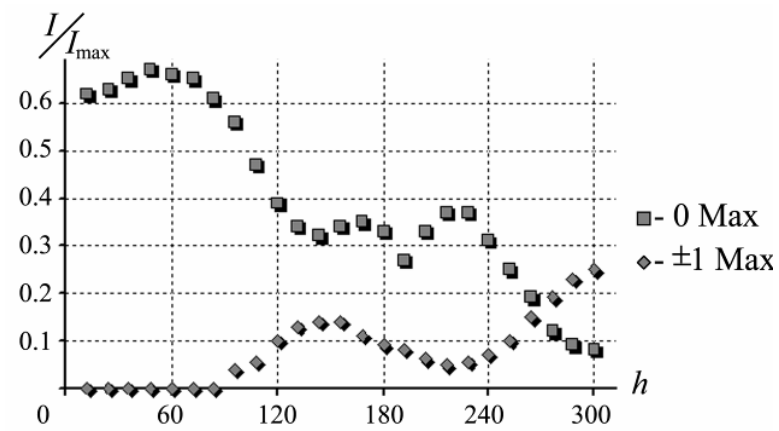

Fig. 3. Intensities of the main $(0 \mathrm{Max})$ and first $( \pm 1$ Max $)$ diffraction orders for various depths of the grating profile.

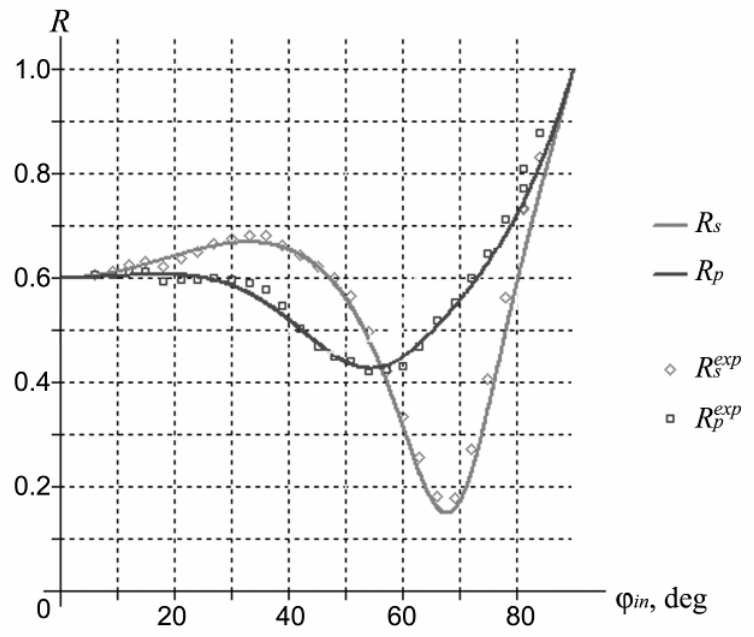

Fig. 4. Experimental and theoretically calculated angular dependences of the reflection coefficient of photoresist surface on the chrome - glass substrate. 
Dependences for orthogonal components of the reflection coefficients for the plane surface of photoresist on the glass substrate with chrome coating $(5 \mu \mathrm{m})$ are shown in Fig. 4 . Taking into account the dependence type and value of reflection coefficients, the refraction coefficient of the photoresist was $n_{P M M A}=$ $1.515+\mathrm{j} 0.0019$ and thickness of the photoresist layer $d_{P M M A}=0.5 \mu \mathrm{m}$. These values were used for determining the effective refraction coefficients of the gratings.

\section{Effective medium model for the grating}

Bruggeman and Maxwell-Garnett effective medium models are based on the Lorentz local field concept [7]. For the Maxwell-Garnett model, the effective permittivity $\varepsilon_{\text {eff }}$ of composite medium that consists of dotted dissemination of material with the permittivity $\varepsilon_{b}=\left(n_{b}+i \kappa_{b}\right)^{2}$ in dielectric matrix $\varepsilon_{m}=\left(n_{m}+i \kappa_{m}\right)^{2}$ is determined as [8]:

$$
\frac{\varepsilon_{e f f}-\varepsilon_{m}}{L \varepsilon_{e f f}+(1-L) \varepsilon_{m}}=\eta \frac{\varepsilon_{b}-\varepsilon_{m}}{L \varepsilon_{b}+(1-L) \varepsilon_{m}},
$$

where $\eta$ is the volume filling factor, $L-$ depolarization factor. The drawback of the Maxwell-Garnett model is the fact that components, which form the nanocomposite, are unequal and the filling factor $\eta<0.3$.

The Bruggeman model is based on the fact that particles with permittivities $\varepsilon_{b}$ and $\varepsilon_{m}$ are placed into medium with the permittivity $\varepsilon_{\text {eff. }}$. Taking into account field averaging within the sample volume, one can receive the following equation for the permittivity: (a)

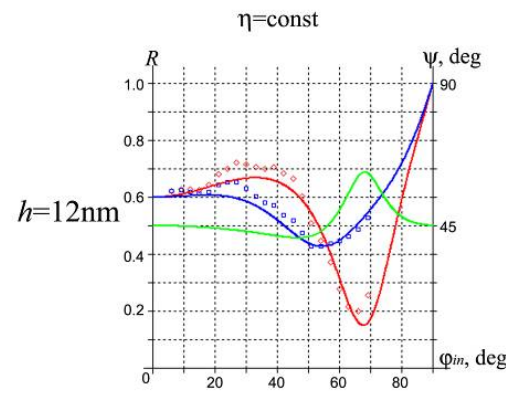

(b)

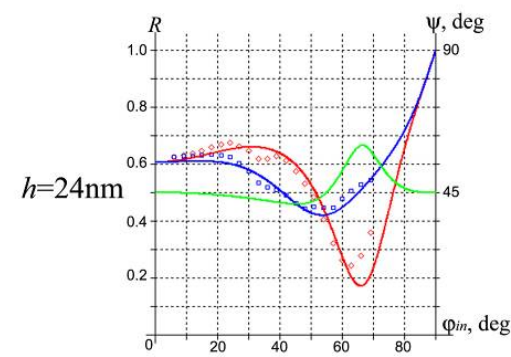

(c)

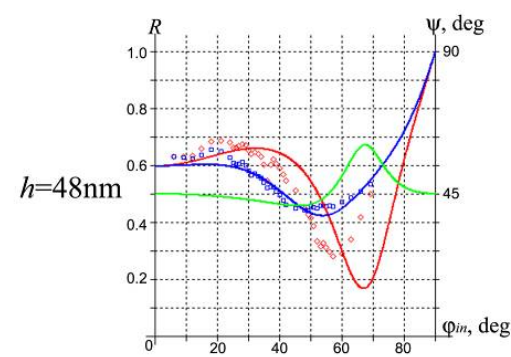

(d)

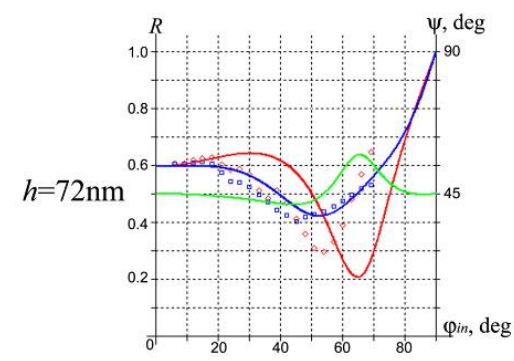

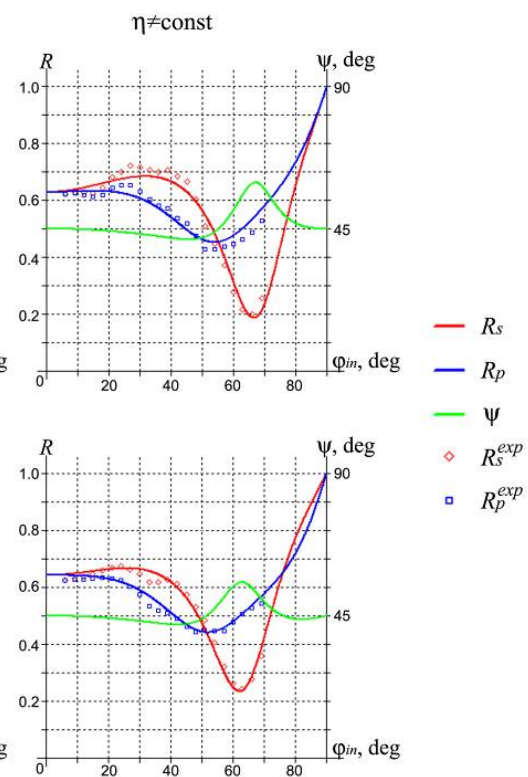
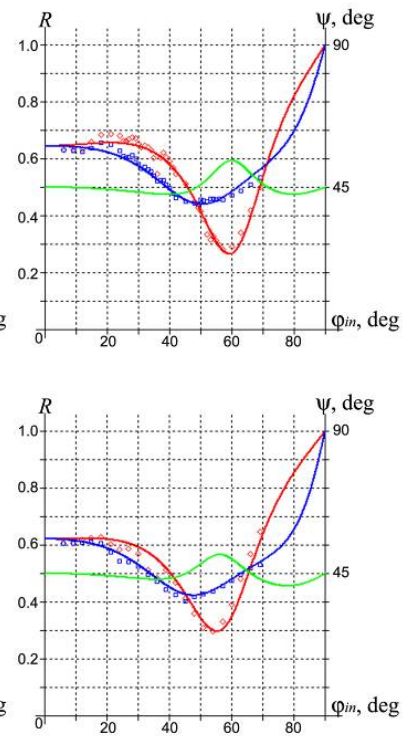

Fig. 5. Angular dependences of the reflection coefficient. 
$\eta_{m} \frac{\varepsilon_{e f f}-\varepsilon_{m}}{L\left(\varepsilon_{e f f}-\varepsilon_{m}\right)+\varepsilon_{m}}+\eta_{b} \frac{\varepsilon_{e f f}-\varepsilon_{b}}{L\left(\varepsilon_{e f f}-\varepsilon_{b}\right)+\varepsilon_{b}}=0$,

where $\eta_{m}+\eta_{b}=1$. It should be mentioned that there is a necessity to consider the filling factors changing in different media for angular investigations of optical illumination characteristics. Calculation of the filling factors is done in the single scattering approximation for some simplification. Thus, taking into account shading the different areas of the grating, the filling factors can be given as:

$$
\begin{gathered}
\eta_{m}^{\text {new }} \approx \frac{\eta_{m}-0.5 n_{m} \sin \varphi_{\text {in }}}{1-0.5\left(n_{m}+n_{b}\right) \sin \varphi_{\text {in }}}, \\
\eta_{b}^{\text {new }} \approx \frac{\eta_{b}-0.5 n_{b} \sin \varphi_{\text {in }}}{1-0.5\left(n_{m}+n_{b}\right) \sin \varphi_{\text {in }}},
\end{gathered}
$$

where $\varphi_{i n}$ is the angle of beam incidence on structured medium. Thus, changing the filling factor should be taken into account when solving the inverse problem to define the effective refraction coefficient.

The influence of the filling factor changing on angular dependences of the reflection coefficient is shown in Fig. 5. As one can see, for the relief depth less than $12 \mathrm{~nm}$, the model of medium with the effective refraction index allows precise description of angular dependences for the reflection index (the error is less than $3 \%$ ). When increasing the diffraction grating relief depth up to $24 \mathrm{~nm}$, the error of the model is increased to $8 \%$. The further increase $(h=24 \mathrm{~nm})$ of the relief depth leads to the error increase up to $15 \%$.

In case of using the variable filling factor, the depth increase up to $72 \mathrm{~nm}$ leads to insignificant error increasing (the error increased from $2 \%$ for $h=12 \mathrm{~nm}$ up to $4 \%$ for $h=72 \mathrm{~nm}$ ). It should be mentioned that usage of variable filling factor allows application of the effective medium model for approximation of angle dependences for heights up to $h=120 \mathrm{~nm}$, although diffraction appears under these conditions (Fig. 3).

\section{Conclusions}

The obtained results show that submicron structural changes of reflective surfaces can be defined by means of multiangular ellipsometry in the optical range. It is shown that multiangular ellipsometry allows determining the changes in the relief height of structured media with the step $12 \mathrm{~nm}$ on the example of light reflection from diffraction gratings. Besides, it has been shown that the model of the effective medium with a variable filling factor allows describing the angular dependences of reflection coefficients with error values less than $5 \%$ for the inhomogeneity heights less than $120 \mathrm{~nm}$. It should be noted that there is some critical height for which application of the medium model with the effective refraction index becomes impossible because of diffraction phenomena.

Development of the offered method can be considered as a perspective way for obtaining the macroscopic characteristics, which describe general properties of investigated materials.

\section{References}

1. S.C. Russev, M.I. Boyanov, J.-P. Drolet, R.M Leblanc, Analytical determination of the optical constants of a substrate in the presence of a covering layer by use of ellipsometric data // $J$. Opt. Soc. Am. A, 16(6), p. 1496-1500 (1999).

2. S.H. Chen, H.W. Wang, T.W. Chang, Absorption coefficient modelling of microcrystalline silicon thin film using Maxwell-Garnett effective medium theory // Opt. Express, 20(S2), p. A197-A204 (2012).

3. J.R. Power, T. Farrell, P. Gerber, S. Chandola, P. Weightman, J.F. McGilp, The influence of monolayer coverages of $\mathrm{Sb}$ on the optical anisotropy of vicinal $\mathrm{Si}(001)$ // Surf. Sci. 372, p. 83-90 (1997).

4. S.A. Kovalenko, M.P. Lisitsa, Thickness dependences of optical constants for thin layers of some metals and semiconductors // Semiconductor Physics, Quantum Electronics \& Optoelectronics, 4(4), p. 352-357 (2001).

5. R.C. van Duijvenbode, E.A. van der Zeeuw, G.J.M Koper, High precision scanning angle ellipsometry // Rev. Sci. Instrum. 72(5), p. 2407-2414 (2001).

6. W. Theiß, Optical properties of porous silicon // Surf. Sci. Repts. 29(3-4), pp. 91-93, 95-192 (1997).

7. W.S. Weiglhofer, A. Lakhtakia, B. Michel, Maxwell-Garnett and Bruggeman formalisms for a particulate composite with bianisotropic host medium // Microwave and Opt. Technol. Lett. 15(4), p. 263-266 (1997).

8. L.A. Golovan, V.Yu. Timoshenko, P.K. Kashkarov, Optical properties of porous-system based nanocomposites // Uspekhi Fizicheskikh Nauk, 177(6), p. 619-638 (2007), in Russian. 